\title{
Resource Allocation Strategies for a Multi Sensor Surveillance System
}

\author{
K Madhava Krishna Henry Hexmoor \\ Dept. of Computer Science and Computer Engineering \\ University of Arkansas \\ Fayetteville, AR 72701 \\ mkrishna@uark.edu \\ hexmoor@uark.edu
}

\begin{abstract}
:
In this paper we expand on our previous efforts $[1,2,3]$ to evaluate strategies for improving tracking performance of a multi-sensor surveillance system. The quality of tracking performance is based on two measures:

(i) The Mean Track Quality (MTM) and

(ii) Total Expected Number of Misses (TEM).

Five strategies of resource allocation were considered based on (a) local versus coordinated, (b) dedicated versus distracted and (c) benevolent. The simulation results suggest that a coordinated and distracted strategy of resource allocation yields the best result throughout while such a strategy coupled with benevolence further improves tracking performance marginally in most cases.
\end{abstract}

\section{Introduction}

A methodology for resource allocation that optimizes tracking performance of a distributed sensor network monitoring multiple moving targets is often a computationally difficult and hard problem to solve. The problem of resource allocation in the case of a multi target surveillance system is classified as an instance of MT-MR-IA/MT-MR-TA type of allocation [4]. MT-MRIA stands for multi-task robots, multi-robot tasks with instantaneous assignment, while MT-MR-TA allows for time extended assignment. The problem can be cast as an instance of the set covering problem (SCP), which is strongly NP hard [5]. The problem is further complicated when the geometry and pattern of the arriving targets is difficult to characterize. Hence in the absence of formal methods of resource allocation, which optimize performance empiric or heuristic methods of evaluating a particular strategy are often resorted to such as in $[6,7]$.

The system considered here is a rectangular surveillance zone with sensors depicted as circles (figure 1). The sensors perform surveillance over the surveillance zone. The surveillance zone is divided into number of square shaped cells as shown in figure 1 for the sake of modeling. The figure shows the sensors placed in their home positions. The radius of vision of the sensor equals the length of the diagonal of the cell.
However the sensor only considers those targets that lie within its four neighboring cells as targets within its field of vision.

Targets crossing the surveillance area are ascribed priorities, which is a fusion of global and local priorities. The global priority for a target is the priority from the point of view of the entire system of sensors. Each robot ascertains its own preference for a target, which is the local priority for that target from the point of view of that sensor. A weighted combination of global and local priorities is used to compute a balanced priority for a target from the point of view of every sensor. Thus, each sensor maintains a list of balanced priorities for every target. The simulation environment used for testing our strategies has been developed through Borland's JBuilder IDE for Java.

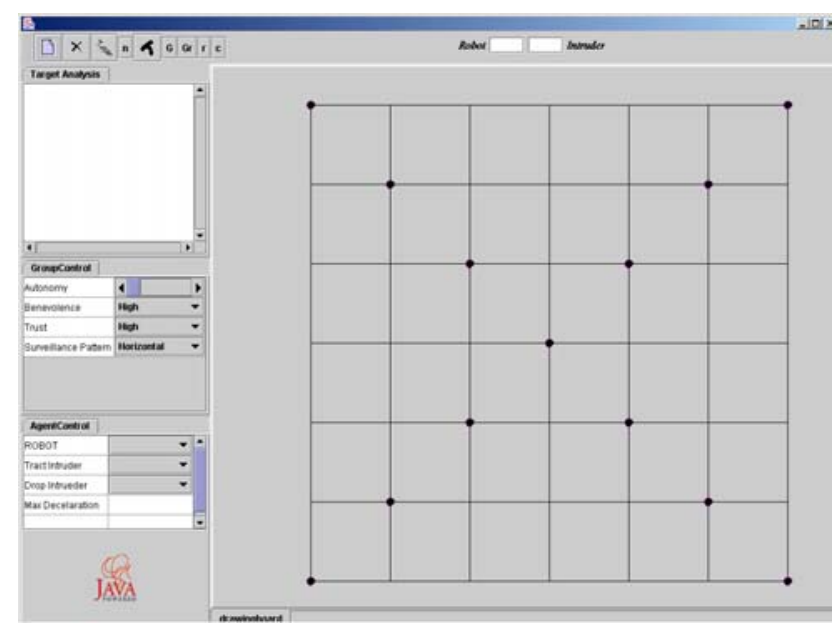

Figure 1. The rectangular surveillance zone with the sensors shown as circles placed along the two zone diagonals at the corners of the cells. The sensors have a radius of vision equal to the diagonal of the cell

Ihis paper presents an empiric classification of strategies for this difficult problem. Different simulation scenarios that cover various possible ways of target entry into the surveillance zone are presented. Under guarantees of ideal sensor characterization of target position, velocities and direction of motion the 
coordinated distracted strategy of resource allocation yields the best performance. This strategy with a mix of benevolence further enhances performance in most cases.

\section{Background Review}

The problem of multi sensor surveillance involves detection of multiple intrusions and/or tracking through coordination between the sensors. Detection and target tracking has been researched from multiple viewpoints. Some efforts have focused on the problem of identifying targets from a given set of data through particle filters [8, and probabilistic methods [9]. The problem of data association or assigning sensor measurements to the corresponding targets were tackled by Joint Probabilistic Data Association Filters by the same researchers such as in [9]. Kluge and others [10] use dynamic timestamps for tracking multiple targets. Krishna and Kalra [11] presented clustering based approaches for target detection and further extended it to tracking and avoidance in [12]. The focus of these approaches has been on building reliable estimators and trackers. They do not use distributed sensors and are not directly useful for the problem of large area surveillance.

In the context of distributed task allocation and sensor coordination Parker [13] proposed a scheme for delegating and withdrawing robots to and from targets through the ALLIANCE architecture. The protocol for allocation was one based on "impatience" of the robot towards a target while the withdrawal was based on "acquiescence". Jung and Sukhatme [14] present a strategy for tracking multiple intruders through a distributed mobile sensor network. Lesser's group have made significant advances to the area of distributed sensor networks [6] and sensor management [15]. In [14] robots are distributed across a region using density estimates in a manner that facilitates maximal tracking of targets in that region. The decision for a robot to move to another region or to stay in its current region is based on certain heuristics. The authors of this paper present their scheme for resource allocation and coordination in a distributed sensor system through a set of fuzzy rules in [1] and further analyze the behavior of system by varying the autonomy of the sensors in [2]. In [3] they present a tracking scheme that guarantees detection of targets to an arbitrary probabilistic accuracy.

\section{Methodology}

The following notations may be useful before embarking on the discussion for ascribing priorities to targets. Let $n_{s}$ represent the total number of sensors and $n_{t}$ the total number of targets in the system. Let $N_{S}$ be the set of all sensors in the system, i.e., $N_{S}=\left\{s 0, s 1, \ldots, s n_{s}\right\}$, where sidenotes the sensor with label $i$. Hence $n_{s}$ is the cardinality of $N_{S}$ or $n_{s}=\left|N_{S}\right|$. In the same vein $N_{T}$ is the set of all targets, $N_{T}=\left\{t 0, t 1, \ldots, t n_{t}\right\}$ and $n_{t}$ is the cardinality of $N_{T}$. We define $S_{t i}$ as the set of all sensors currently monitoring target $t i$ and $T_{s i}$ as the set of all targets being monitored by sensor $s i$. Then $\bar{S}_{t i}$ is the set of all sensors currently not detecting $t i$. We denote the number of samples a target $t i$ is likely to be detected or observed by sensor $s j$ by $o_{t i s j}$, where $s j \in S_{t i}$. If the time for which $t i$ is likely to be within the field of vision of $s j$ is $t_{t i s j}$ and the sampling interval is $d t$ then $o_{t i s j}=t_{t i s j} / d t$. The sampling time indicates the time interval between two successive scans of the environment by the sensors. $S F_{t i}$ is the set of all sensors that are likely to detect $t i$ in the future though they are not observing it currently. Hence $S F_{t i} \subset \bar{S}_{t i}$.

\subsection{Priority ascription to targets}

Sensors reason about targets by ascribing priorities to them. Priorities serve as handles that aid in decision making. Whenever a sensor detects a target it updates information about the target regarding its current position, velocity and motion direction to a whiteboard. The whiteboard is a common data store of resources that includes public methods and variables, which can be modified and accessed by other programs of the project. Other sensors can come to know about this target by accessing these variable or invoking methods from this common pool.

Every target is given a global priority that portrays the priority to that target from the point of view of the entire sensing apparatus.

Ascribing global priority:

The global priority for a target $t i$ is determined by three parameters namely:

- $\quad p 1_{t i}$ : The maximum number of times target $t i$ is likely to be further observed by one of the sensors currently monitoring it. In other words $p 1_{t i}=\max _{s j}\left(o_{t i s j}\right) \forall s j \in S_{t i}$ 
- $\quad p 2_{t i}$ : The number of sensors currently not detecting $t i$ but are expected to detect it in future. $p 2_{t i}=\left|S F_{t i}\right|$

- $\quad p 3_{t i}$ : The measure of possibility that sensors in $S F_{t i}$ would be in a position to monitor $t i$ in the future. This is elaborated in the subsequent section on sensor coordination. $p 3_{t i}=\sum_{s j} m_{t i s j} \forall s_{j} \in S F_{t i}$, where $m_{t i s j}$ is the measure of the possibility of $s j$ to take care of $t i$

The fuzzy rulebase that infers the global priority $g p_{t i}$ for any target in the system is tabulated in table 1. For notational convenience we remove the suffix $t i$ associated with the parameters henceforth.

The membership functions associated with the antecedent and consequent variables are not shown here due to brevity of space and since they are not what that constitute the main thrust of this effort.

\begin{tabular}{|c|c|c|c|}
\hline $\mathbf{p 1}$ & $\mathbf{p 2}$ & $\mathbf{p 3}$ & $\mathbf{g p}$ \\
\hline $\mathrm{H}$ & $\mathrm{L}$ & $\mathrm{X}$ & $\mathrm{M}$ \\
\hline $\mathrm{H}$ & $\mathrm{M}$ & $\mathrm{X}$ & $\mathrm{LM}$ \\
\hline $\mathrm{H}$ & $\mathrm{H}$ & $\mathrm{X}$ & $\mathrm{L}$ \\
\hline $\mathrm{L}$ & $\mathrm{L}$ & $\mathrm{X}$ & $\mathrm{H}$ \\
\hline $\mathrm{L}$ & $\mathrm{M}$ & $\mathrm{X}$ & $\mathrm{HM}$ \\
\hline $\mathrm{L}$ & $\mathrm{H}$ & $\mathrm{X}$ & $\mathrm{M}$ \\
\hline $\mathrm{H}$ & $\mathrm{X}$ & $\mathrm{H}$ & $\mathrm{L}$ \\
\hline $\mathrm{H}$ & $\mathrm{X}$ & $\mathrm{L}$ & $\mathrm{LM}$ \\
\hline $\mathrm{L}$ & $\mathrm{X}$ & $\mathrm{H}$ & M \\
\hline $\mathrm{L}$ & $\mathrm{X}$ & $\mathrm{L}$ & $\mathrm{H}$ \\
\hline
\end{tabular}

Table 1. Fuzzy rulebase for global priority inference. The linguistic labels for the fuzzy sets are: $\mathrm{H}=$ high, $\mathrm{L}=$ low, $\mathrm{M}=$ medium. $\mathrm{LM}=$ low medium and $\mathrm{HM}=$ high medium

\section{Ascribing local priority:}

Every target $t i$ is associated a local priority from the point of view of every sensor $s i$ in $N_{S}$. The parameteric basis for local priority computations vary marginally with regard to whether $s i$ belongs to $S_{t i}$ or not. If $s i \in S_{t i}$ the local priority is based on the time for which $s i$ would have to track $S_{t i}$ before another sensor engages $t i$. If $s i \notin S_{t i}$ the computation is based on the time for which $s i$ would have to wait for $t i$ before $s i$ can engage $t i$. The local priority for a target $t i$ from the point of view of a sensor $s j$ is denoted as $l p_{s j}$. Denoting either of the times as $t_{\text {wait }}$ the rulebase for computing local priority is tabulated in table 2 where the symbols carry the same linguistic labels as in table 1.

\begin{tabular}{|c|c|}
\hline$t_{\text {wait }}$ & $l p_{s j}$ \\
\hline $\mathrm{L}$ & $\mathrm{H}$ \\
\hline $\mathrm{M}$ & $\mathrm{M}$ \\
\hline $\mathrm{H}$ & $\mathrm{L}$ \\
\hline
\end{tabular}

Table 2. Fuzzy rulebase for local priority inference.

\section{Ascribing balanced priority:}

The balanced priority for $t i$ from the point of view of $s j$ is obtained by fusing local and global priorities as follows:

$b p_{s j}=w_{g^{\prime}}(g p)+\left(1-w_{g^{\prime}}\right) l p_{s j}$, where

$w_{g^{\prime}}=w_{g}\left(1-w_{a b}\right)$. Here $w_{g}$ takes unitary value if $s j \in S_{t i}$, else its value decreases linearly with the time taken by $t i$ to enter the field of vision of $s j . w_{a b}$ represents the autonomy bias of a sensor towards its own preference for the target [local priority] rather than the preference as ascribed to the target by the entire system [global priority]. Under high values of $w_{a b}$ the balanced priority would reflect the individual sensor's preference for the target more than the global preference.

\subsection{Resource Allocation}

Resource allocation is the problem of allocating a sensor to a target. Let the target with the highest balanced priority for a sensor $s j$ be denoted as $t_{s j}^{m}$ and the sensor which would detect a target $t i$ for maximum number of times amongst the set of sensors currently observing it be represented as $s_{t i}^{m}$. The superscript $\mathrm{m}$ indicates maximum.

\section{Local versus Coordinated}

In the local scheme the resource allocation simply assigns the sensor to the target that has the highest balanced priority, i.e. $s j$ is allocated to $t_{s j}^{m}$.

In the coordinated scheme when two sensors compete for the same target that sensor is assigned to the target that can see the target for a longer duration from its current location. The unallocated sensor is assigned to the 
next highest priority target from its list for which there is no other competing sensor that will see the target longer.

There are also other rules for resource allocation in the coordinated scheme that take into account visibility relation between sensors and targets and the geometry of the expected sensor tracks before making the final allocation [1].

\section{Dedicated versus Distracted}

In the dedicated strategy the sensor does not reassign itself to the target with the highest priority provided it has not completed tracking the previous target for a certain duration of samples

In the distracted strategy the sensor switches to the target with highest priority as soon as it senses one.

\section{Benevolence:}

When sensors happen to come together and if their assigned targets move nearly along same directions one of the sensors alone continues to monitor the target while the remaining ones return to their home position. The sensor that continues to track is the one for which the assigned target has the highest magnitude of priority when compared with the assigned targets of other sensors that return to their home position.

\section{Performance Measures}

The following parameters are used to measure the performance of the system.

$$
\text { - Mean Tracking Quality } M T Q=\sum_{i} \frac{d(t i)}{N} \text {, }
$$
where, $d(t i)$ is the number of detections of the target $t i$ and $N$ is a normalization constant. $N$ is the minimum number of times a target needs to be detected for a sufficient characterization of it. $N$ is fixed at 100 in all the examples discussed in this paper. $M T Q$ can have a value greater than unity and higher the tracking quality the performance is considered better

- Total expected number of misses, $T E M=\sum E M(s j)$. Here $E M(s j)$ represents the expected number of targets that will go undetected by $s j$ during the time $s j$ is in pursuit of some other target. Lower the value of TEM, better is the tracking performance.
The computation of $T E M$ is not straightforward. Targets are assumed to come at rate $\lambda$ in the space occupied between any two of the vertices formed by the meeting point of the rows and columns on the boundary. For a surveillance zone such as in figure 1 consisting of six rows and columns targets enter at rate $\lambda$ at six of those spaces formed between the points of intersection of the rows and columns on the boundary. Hence from each boundary of the rectangle the rate of entry is $6 \lambda . \lambda$ is fixed at 0.1 for all the examples discussed in this paper. Then the apparent rate at which each sensor would see a target, $\lambda_{S J}$ provided it is stationary is given by the following approximation:

$\lambda_{S J}=\lambda \sum_{k=1}^{P} \frac{\theta_{k}}{\pi}$, where, $\theta_{k}$ is the angle subtended from the point where the target enters the boundary at the home position of sensor $s j$. Since the entry points of the arriving targets are not known a-priori, $\theta_{k}$ is computed assuming that the target arrives at the midpoint of the region between the intersection of rows and columns along the perimeter of the surveillance zone. In the figure below (figure 4) the targets are assumed to enter at points p1, p2, p3, ... along the perimeter of the surveillance zone. For the sensor centered at ' $b$ ', the angle subtended by the target entering at $\mathrm{p} 4$ is shown marked $\theta$. This angle covers the span of all the targets that will cross the region of surveillance of the sensor at ' $b$ ' by agents entering at $\mathrm{p} 4$. The total span of the angle for a target entering at all those points is $\pi$ radians or in other words all targets that enter the surveillance zone have to necessarily be within a span of $\pi$ radians from the point of entry for them to be within the surveillance zone.

Let $T$ be the time for which a sensor $s j$ is away from its home position in pursuit of a target. We then compute the apparent time $T_{a}$, the time for which a target that would have been in the field of vision of $s j$ had $s j$ been stationary at its home position perceives $s j$ to be away. A round path of the sensor from its home position and back is a composition of several piecewise linear segments. If one such path consists of $P$ number of such segments then the apparent time for which the sensor is away is given by: $t_{a p p}^{i}=\frac{d_{a p p}^{i}}{v_{s}}$, assuming constant velocity of the sensor $v_{s}$. Then $T_{a}$ is a summation over all such segments and is given by 
$T_{a}=\sum_{i} t_{a p p}^{i}$. The apparent distance for which the sensor has moved away over one segment is

$$
d_{a p p}^{i}=\left\{\begin{array}{c}
\int_{s 1}^{s 2} \frac{s . d s}{D_{f}} ;\left|d\left(p_{1}\right) \leq D_{f}\right|,\left|d\left(p_{2}\right) \leq D_{f}\right| \\
\int_{s 1}^{D_{f}} \frac{s . d s}{D_{f}}+s 2-D_{f} ;\left|d\left(p_{1}\right) \leq D_{f}\right|,\left|d\left(p_{2}\right)>D_{f}\right| \\
s 2-s 1 \quad ;\left|d\left(p_{1}\right)>D_{f}\right|,\left|d\left(p_{2}\right)>D_{f}\right|
\end{array}\right.
$$

Here $p 1, p 2$ are the positions of the two endpoints of the segment measured with respect to some reference frame and $d(p 1)=s 1$ and $d(p 2)=s 2$ are the distances of those locations from the home position of the sensor. $D_{f}$ is the distance at which the sensor leaves its home area completely unguarded. If $T_{\text {esc }}$ represents the average time for which a sensor should be away such that a target can get past it original covering area completely undetected, the expected number of targets that would be missed by the sensor is given by the approximation $\lambda_{S J} \frac{T_{a}}{T_{\text {esc }}}$. In other words the final expression states if $\lambda_{S J}$ is the average rate of entry within the original covering area of $s j$ and if $s j$ is away from the point of view of the entering target for a time $T_{\text {esc }}$ (i.e., $T_{a}=T_{\text {esc }}$ ) then $\lambda_{S J}$ number of targets can be expected to go undetected on an average. $T_{e s c}$ is fixed as the time taken by a target to move from one vertex to its opposite vertex along the main diagonal of the covering area.

\section{Simulation Results}

The simulations present results from five different scenarios. Table 3 tabulates the performance. The rows denote the kind of resource allocation strategy used while the main columns denote the five test scenarios. Each main column of the scenario is divided into two sub columns for the performance measures $M T Q$ and TEM. The results indicate that as far as $M T Q$ is concerned there is no clear-cut demarcation between strategies. However the coordinated distracted strategy yields the least TEM in all the cases except in scenario 3, where the distracted and dedicated versions of the coordinated strategy are on par. Also the coordinated distracted benevolent scheme further improves the performance of TEM by lowering it in three of the five scenarios. A ranking of the resource allocation strategy would take the form coordinated distracted benevolent $>$ coordinated distracted $>$ coordinated dedicated $>$ local distracted $>$ local dedicated. The symbol $>$ is read as "better than".

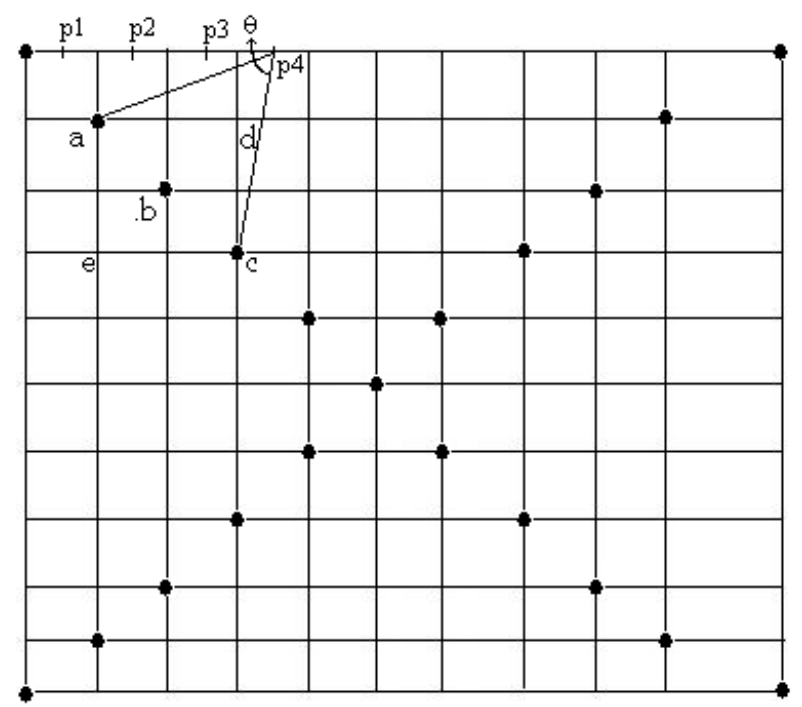

Figure 2: Targets are modeled as entering at locations p1, $\mathrm{p} 2, \mathrm{p} 3, \ldots$, as shown. These are the midpoints of the space between the meeting points of the columns and rows along the perimeter

The scenarios used in simulations are based on various possible modalities of target entry into the surveillance zone. Test scenario 1 involves 20 target intrusions in random directions, five from each boundary. Scenario 2 considers 20 targets introduced in parallel from left to the top. Scenario 3 considers two sets of targets introduced in parallel from left to right separated by time intervals, while 4 involves 2 sets of 10 targets introduced from left to top and top to right along directions such that there is a cluttering of targets at top right and bottom right areas of the zone. Scenario 5 involves 30 targets introduced randomly in various directions.

Figures $3 \mathrm{a}$ and $3 \mathrm{~b}$ depict the final snapshots when all the targets have crossed the zone for the coordinated distracted and local dedicated strategies for scenario 3, while figures $4 \mathrm{a}$ and $4 \mathrm{~b}$ are the snapshots for the same strategy for scenario 1 . It is seen that the overall movement of the sensors is less in the coordinated distracted strategy vis-à-vis local dedicated strategy though the $M T Q$ for both the strategies are not vastly different for both scenarios as tabulated in table 3 . 


\begin{tabular}{|l|c|c|c|c|c|c|c|c|c|c|}
\hline \multirow{2}{*}{ Strategy } & \multicolumn{2}{|c|}{ Scenario 1 } & \multicolumn{2}{c|}{ Scenario 2 } & \multicolumn{2}{c|}{ Scenario 3 } & \multicolumn{2}{c|}{ Scenario 4 } & \multicolumn{2}{c|}{ Scenario 5 } \\
\cline { 2 - 13 } & $M T Q$ & $T E M$ & $M T Q$ & $T E M$ & $M T Q$ & $T E M$ & $M T Q$ & $T E M$ & $M T Q$ & $T E M$ \\
\hline Coordinated Distracted Benevolent & 0.49 & 18 & 0.73 & 8 & 0.91 & 7 & 0.71 & 10 & 0.61 & 14 \\
\hline Coordinated Distracted & 0.49 & 18 & 0.72 & 11 & 0.91 & 11 & 0.71 & 13 & 0.61 & 11 \\
\hline Coordinated Dedicated & 0.51 & 27 & 0.74 & 17 & 0.95 & 11 & 0.78 & 23 & 0.61 & 38 \\
\hline Local Distracted & 0.49 & 46 & 0.81 & 48 & 1.00 & 43 & 0.77 & 51 & 0.6 & 29 \\
\hline Local Dedicated & 0.51 & 44 & 0.82 & 56 & 0.93 & 41 & 0.75 & 65 & 0.58 & 44 \\
\hline
\end{tabular}

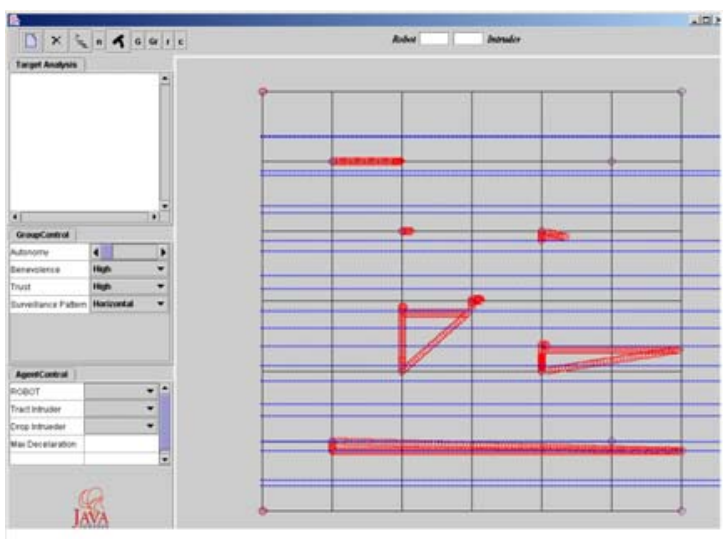

Figure 3a: The final snapshot of the coordinated distracted strategy for scenario 3 . Sensor paths shown in red, targets in blue

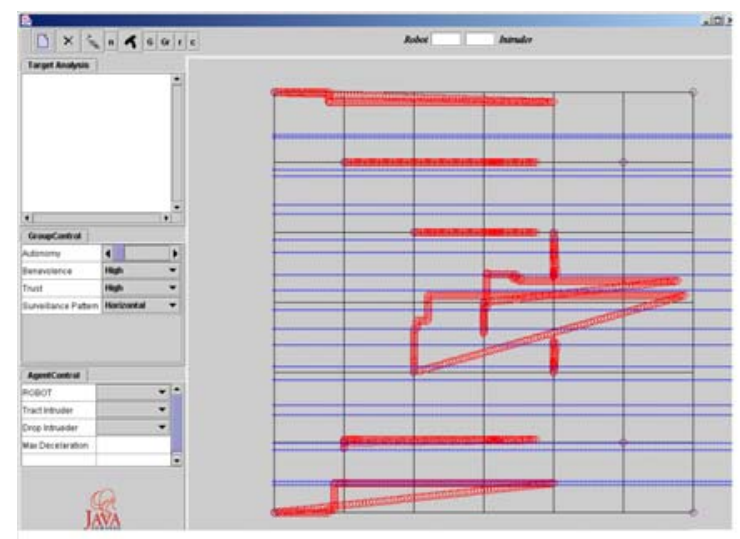

Figure 3b: The final snapshot of the local dedicated strategy for scenario 3

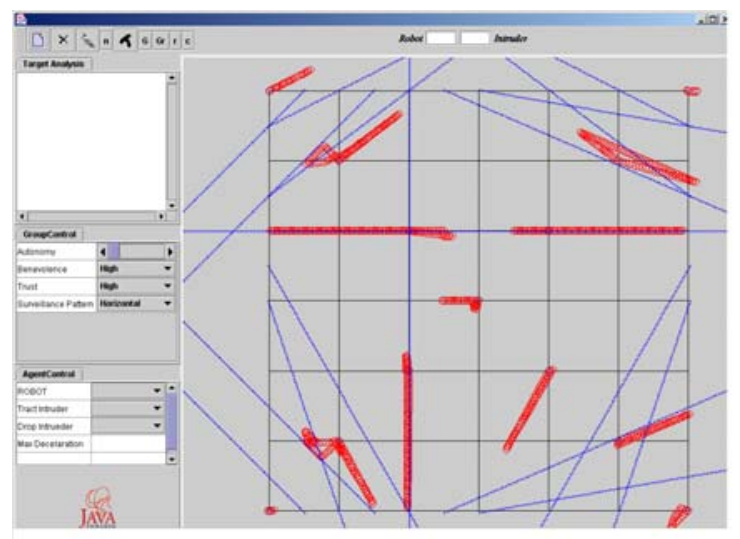

Figure 4a: The final snapshot of the coordinated distracted strategy for scenario 1.

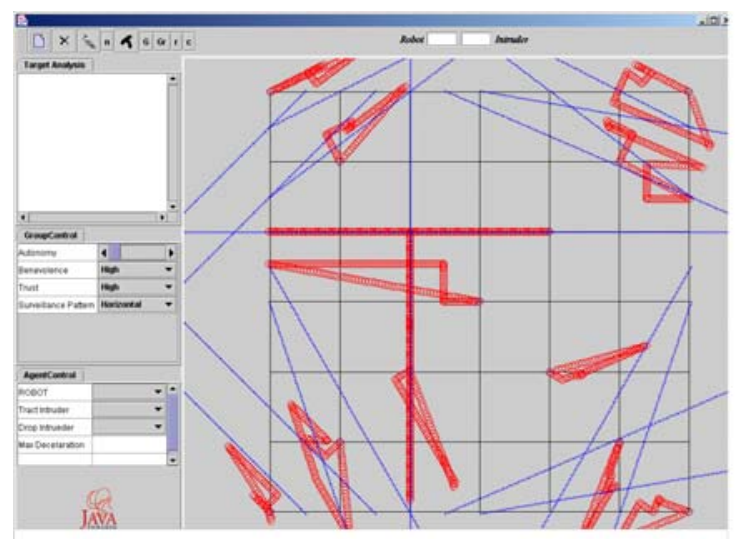

Figure 4b: The final snapshot of the local dedicated strategy for scenario 1 


\section{Discussion}

The obvious questions to be raised with such an approach are whether the test scenarios are representative and cover all possible modalities of target entry and if the ranking of resource allocation mechanisms identified here can be generalized. The answer to this is that as with any empiric study the results are suggestive or indicative than completely conclusive. However the results obtained can be explained in a qualitative fashion.

The coordinated scheme has better TEM than the local method since the allocation mechanism prevents sensors from tracking the same target thereby widening coverage. Also the geometric considerations involved in the coordinated approach assigns the target to that sensor that can track it longer without leaving its home position among the sensors that bid for that target. Hence widening of coverage area and retention of the sensor at its home position contribute to the lower TEM in the coordinated approach.

The distracted modality results in frequent change of targets. If the sensor has not left its home position completely then it is most likely to get attracted to a new target from one of the boundaries of the home area. Hence in the distracted mode the sensor is more likely to loiter in the home area while a dedicated pursuit of a single target guarantees definite departure from the home area. A sensor that is mobile but scattered in its home area has lesser chances of missing targets than that has completely left its home area.

\section{Conclusions:}

An empiric verification of various resource allocation strategies for a mobile multi sensor surveillance system has been presented. Among the five different modalities of sensor allocation the coordinated distracted benevolent strategy was found to be the most suitable. All the strategies gave similar mean tracking qualities. However in reducing targets that go past unnoticed the coordinated distracted modality yields the best performance. A qualitative discussion that analyzes the reasons for the observed performance is also presented. As with any empiric study the results are suggestive and indicative and present a general guideline for the difficult problem of resource allocation of sensors to targets such as in a surveillance system. These results would be useful for any kind of multi sensor system that finds various applications such as border surveillance, tracking and search and rescue. A formal method for obtaining a desired TEM performance within probabilistic guarantees is presented by the authors in [3].

\section{References:}

[1] K Madhava Krishna, H Hexmoor and S Pasupuleti, "A Surveillance System Based on Multiple Mobile Sensors", Proceedings of FLAIRS, 2004, Special Track in Sensor Fusion.

[2] K Madhava Krishna, H Hexmoor and S Pasupuleti, "Role of Autonomy in a Distributed Sensor Network", Proceedings of ICAI, 2004

[3] K Madhava Krishna and Henry Hexmoor, "A Framework for Measuring Tracking Performance of a Sensor Network", to appear in Proceedings of PerMIS, 2004 (Performance Metrics in Intelligent Systems)

[4] Brian Gerkey and Maja Mataric, "A formal analysis and taxonomy of task allocation in multi-robot systems", to appear in the Intl. Journal of Robotics Research

[5] Korte, B. and Vygen, J., Combinatorial Optimization: Theory and Algorithms, Springer Verlag, Berlin

[6] B Horling, R Vincent, R Miller, J Shen, R Becker, K Rawlins, and V Lesser, "Distributed Sensor Network for Real Time Tracking", In Proceedings of the 5th International Conference on Autonomous Agents:. 417-424, 2001.

[7] Maja Matric, Gaurav Sukhatme and Esben Ostergaard, "Multi-robot Task Allocation in Uncertain Environments", Autonomous Robots 14, 255-263, 2003

[8] D Schulz; W Burgard, D Fox and A Cremers, "Tracking multiple moving targets with a mobile robot using particle filters and statistical data association", IEEE International Conference on Robotics and Automation, 1165-1170, 2001

[9] D Schulz and W Burgard, "Probabilistic state estimation of dynamic objects with a moving mobile robot", Robotics and Autonomous Systems, 2001.

[10] B. Kluge, C Kohler and E Prassler, "Fast and robust tracking of multiple objects through a laser range finder", IEEE International Conference on Robotics and Automation, 11651170,2001

[11] K Madhava Krishna and P K Kalra, "When does the robot perceive a dynamic object", Journal of Robotic Systems, 19(2), 2002

[12] K Madhava Krishna and P K Kalra, "Detection tracking and avoidance of multiple dynamic objects", Journal of Intelligent and Robotic Systems, 33(4): 371-408, 2002

[13] L Parker, "Cooperative robotics for multi-target observation", Intelligent Automation and Soft Computing, $5[1]: 5-19,1999$

[14] B Jung and G.S. Sukhatme, "Multi-Target Tracking using a Mobile Sensor Network", Proc., IEEE Intl. Conf. On Robotics and Automation, 2002 
[15] B Horling, R Miller, M Sims, and V Lesser, "Using and Maintaining Organization in a Large-Scale Distributed Sensor Network", In Proceedings of the Workshop on Autonomy, Delegation, and Control, (AAMAS 2003). 